\title{
CARACTERIZACIÓN DE LA PLANEACIÓN Y EL CONTROL DE LA PRODUCCIÓN EN LAS MIPYMES DEL SECTOR MANUFACTURERO EN LA CIUDAD NEIVA
}

\section{CHARACTERIZATION OF PLANNING AND PRODUCTION CONTROL PROCESSES IN MSMES OF THE MANUFACTURING SECTOR IN NEIVA}

\author{
Oscar Jaramillo O.*, Álvaro Tejada O..*, Alonso Clavijo G. ${ }^{* *}$
}

\begin{abstract}
Resumen
El estudio realizado en las Mipymes de Neiva para la caracterización de la Planificación y el control de la producción, los cuales se constituyen en pilar fundamental para alcanzar los niveles de competitividad regional se basó en los estudios de tiempos y movimientos, capacidad de producción, eficiencia de cada centro de producción y del total de la planta, nivel de utilización de cada centro, y del total de la planta, registros de ventas de los últimos cinco años y la técnica del pronóstico para encontrar el tamaño de las ventas a futuro. Para la planeación a corto plazo se estudiaron los siguientes aspectos: Plan Maestro de Producción, evaluación de los diferentes planes, capacidad necesaria de cada lote de producción, diagramas de ruta para sus productos, registros de carga de cada operación, lista de materiales para cada producto, registro para identificar cuando pedir y registro de inventarios. El estudio arroja como resultado que las pequeñas empresas y las micro empresas, carecen de sistemas de planeación y control y que los registros históricos necesarios para adelantar dichas labores son incipientes, con baja utilización de mano de obra calificada y poca intervención de profesionales en los procesos de gestión empresarial.
\end{abstract}

Palabras clave: Planificación, control de producción, competitividad.

\section{Abstract}

The study conducted in Neiva MSMEs for characterizing of planning and production control, which constitute the cornerstone for achieving regional competitiveness levels was based on time and motion studies, production capacity, efficiency of each production center and overall efficiency of the plant, the level of use of each production center and the level of use of the whole plant, sales records of the last five years, and the forecast technique to find the size of future sales. For short-term planning the following aspects were studied: Master Plan of Production, evaluation of different plans, required capacity of each production lot, route diagrams for products, records of load of each operation, bill of materials for each product, registers to identify when to order and inventory records. The study showed as a result that small enterprises and micro enterprises lack of planning and control systems and that the historical records required for these tasks are emerging, with low utilization of skilled labor and little intervention of professionals in business management processes.

Key words: planning, production control, competitiveness

Artículo recibido: 13/01/2013 Aprobado: 22/07/2013

* Ingeniero Industrial. Profesor Corporación Universitaria del Huila Corhuila. Email: osjaramilloc@gmail.com

** Ingeniero Industrial. Profesor Corporación Universitaria del Huila Corhuila. Email: alvaro.tejada@corhuila.edu.co

*** Ingeniero Químico. Profesor Corporación Universitaria del Huila Corhuila. Email: alcla123@yahoo.com 


\section{Introducción}

La productividad y confiabilidad de las empresas solo se logra mediante una planeación adecuada de los recursos que permita responder de manera acertada a los cambios del mercado, esa capacidad de respuesta es la que nos permite medir el grado de competitividad de las organizaciones. Para la elaboración de planes de producción ajustados a las necesidades, las organizaciones deben contar con una cultura que les permita mantener información confiable sobre los costos, la capacidad de los recursos disponibles, indicadores de eficiencia y utilización y establecer políticas que les permitan evaluar el desarrollo y la efectividad de estos. Esas entonces son las variables que nos permiten caracterizar los sistemas de planeación y control de las empresas del sector manufacturero de la ciudad.

El principal interés de este trabajo es determinar las características que distinguen la administración y planeación de los sistemas productivos de las pymes de la ciudad de Neiva, mediante el conocimiento de la estructura y el funcionamiento de sus sistemas de planificación y control de la producción; identificando si cuentan con sistemas de información, planeación y control adecuados y confiables y con las técnicas y herramientas adecuadas para la toma de decisiones en sus procesos.

Las pequeñas y medianas empresas (PYMES) representan el $96 \%$ de las empresas en Colombia, el $25 \%$ del PIB, el $25 \%$ de las exportaciones y generan el $49,6 \%$ del empleo industrial. Estas empresas frecuentemente tienen problemas en cuanto a competitividad, calidad y $\operatorname{costos}^{1}$. De acuerdo con la oficina de Desarrollo Empresarial de la Cámara de Comercio de Neiva² a junio de 2010 se encontraban registradas 7516 PYMES clasificadas según la Ley 905 de 2004, de las cuales dos correspondían a medianas empresas, 20 a pequeñas empresa y 7494 a microempresas. De acuerdo con los datos de la Cámara de Comercio de Neiva, la mayor cantidad de empresas que se constituyen se orientan a desarrollar sus actividades en el sector comercio con una participación del 51\%, seguida de hoteles y restaurantes con el $12 \%$, manufactura $8 \%$, construcción $3 \%$, agropecuario $2 \%$ y el resto en otros sectores.
La participación sectorial en la inversión que se mide por medio de los activos de las empresas es diferente; las actividades de comercio participan con el $33 \%$, construcción con el $13 \%$, manufactura con el $6 \%$, hoteles y restaurantes con el $2 \%$, agropecuario $2 \%$, pesca $1 \%$ y la otra inversión en el resto de sectores (Ver figura 1).

La competitividad de una empresa se mide por el conocimiento que esta tenga de lo que es capaz de hacer (El cliente puede suministrar la información necesaria sobre lo que desea o la empresa cuenta con los diseños, tolerancias, especificaciones, lista de partes y especificaciones de calidad), cuándo es capaz de hacerlo, (Puede establecer fecha de entrega de los pedidos), qué herramientas son necesarias para su elaboración (Se inspeccionan las herramientas, los equipos necesarios antes de realizar el trabajo en el taller) y cómo lo haremos (Podemos determinar antes de empezar la producción, cómo se va hacer el producto, las hojas de ruta 0 instrucciones de los procesos, etc) ${ }^{3}$.

La prioridad para los entes gubernamentales que tienen que ver con las políticas de competitividad y desarrollo de las regiones y de las asociaciones de empresarios establecidas en la región es y debe ser conocer cuál es la capacidad instalada de cada uno de los sectores en que se encuentran dividida la manufactura, establecer cuál

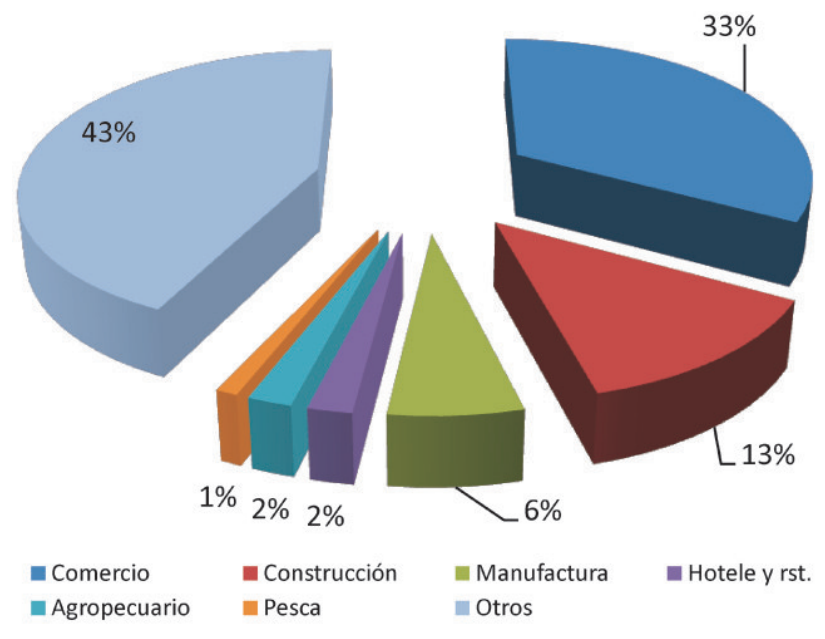

Figura 1. \% de participación por sectores según cantidad de activos

1. PYME: Balance 2005 - Perspectivas 2006 - TLC- Negociación de salario mínimo. Acopi Regional Bogotá. Fecha de consulta: abril 2007. Disponible en: http://www.acopibogota.org.co

2. Informe MOVISOC 2010 Cámara de Comercio de Neiva. Disponible en: www.ccneina.org.

3. Control adecuado de la producción - Allen B. Dickeman disponible en: http://www.control adecuado de la producción 
es la capacidad de respuesta de las organizaciones en caso de requerirse un mayor volumen de sus productos; identificar la tecnología aplicada; conocer sus desarrollos en investigación y establecer cuál es la propuesta de nuevos productos o de mejoras de los que tienen en el mercado; identificar cuáles son las estrategias para reducir los costos operacionales y si se cuenta con la tecnología de punta parta el desarrollo de los procesos y con el personal adecuado para tal fin.

La pregunta problema es: ¿De qué manera planifican y controlan la producción las Mipymes de la ciudad de Neiva y qué herramientas informáticas utilizan?

De acuerdo a consultas efectuadas a las universidades de la región que cuentan con facultades de ingeniería industrial o de administración de empresas y en la Cámara de Comercio de Neiva no se encontró trabajo de investigación referente al tema de este trabajo.

\section{Metodología}

El estudio se inicia recolectando información sobre el número de empresas de la ciudad, direcciones, sector económico al que pertenecen y un resumen de su actividad manufacturera. Se selecciona la información de acuerdo al CÓDIGO INDUSTRIAL INTERNACIONAL UNIFORME (CIIU) y se obtiene el siguiente resultado:

En la Ciudad de Neiva se encuentran 12 unidades empresariales que están dedicadas al sector A, Agricultura, Caza, Pesca y Silvicultura, 9 de ellas en el sub sector producción específicamente agrícola y 3 en el sector pecuario (Ver figura 2); en el subsector B Pesca no se encuentra registrada ninguna empresa; en el subsector C Explotación de minas y canteras se encuentran 9 empresas todas en el subsector Extracción de piedra, arena, arcillas, cal, yeso, caolín y bentonitas (Ver figura 3).

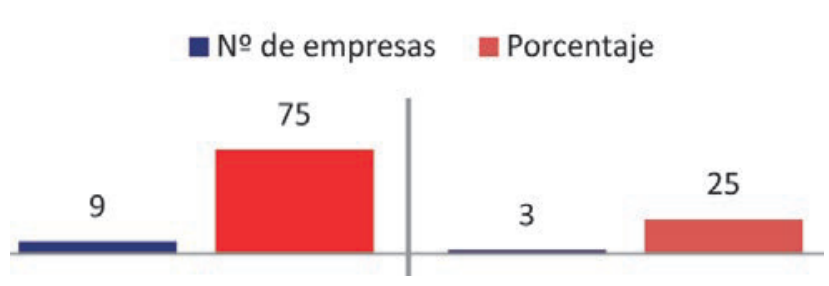

Figura 2. Empresas del subsector A. Agricultura, ganadería, caza y pesca

№ de empresas $\square$ Porcentaje

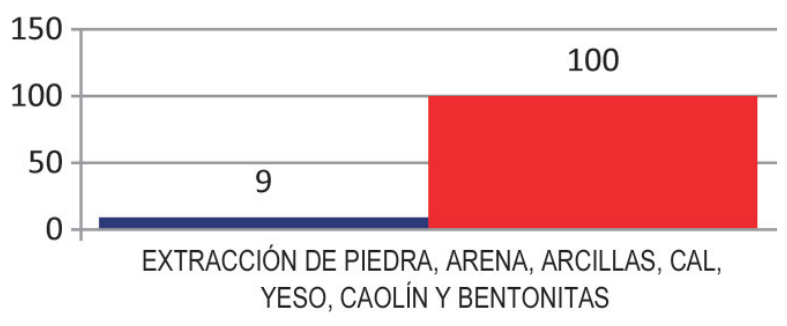

Figura 3. Número de empresas en cada subsector del sector $\mathrm{C}$. Explotación de minas y canteras

En el sector D Industrias manufactureras se encuentran 718 empresas divididas en la mayoría de los subsectores. Los subsectores más representativos 0 donde mayor número de empresas se encuentran son: Elaboración de productos alimenticios y bebidas con 239 empresas que representan el $33 \%$ del total, el sector de fabricación de prendas de vestir; preparado y tejido de pieles aglutina 126 empresas que representan el $18 \%$ del total de empresas del sector. En el sector Fabricación de productos elaborados de metal, excepto maquinaria y equipo, se agrupan 94 empresas que representan el 13 del total. En los sectores Fabricación de muebles; industrias manufactureras ncp y Actividades de edición e impresión y de reproducción de grabaciones se agrupan: 63 unidades que corresponden al $9 \%$ y 72 empresas al $10 \%$ del total respectivamente, (ver figura 4 y tabla 1 ).

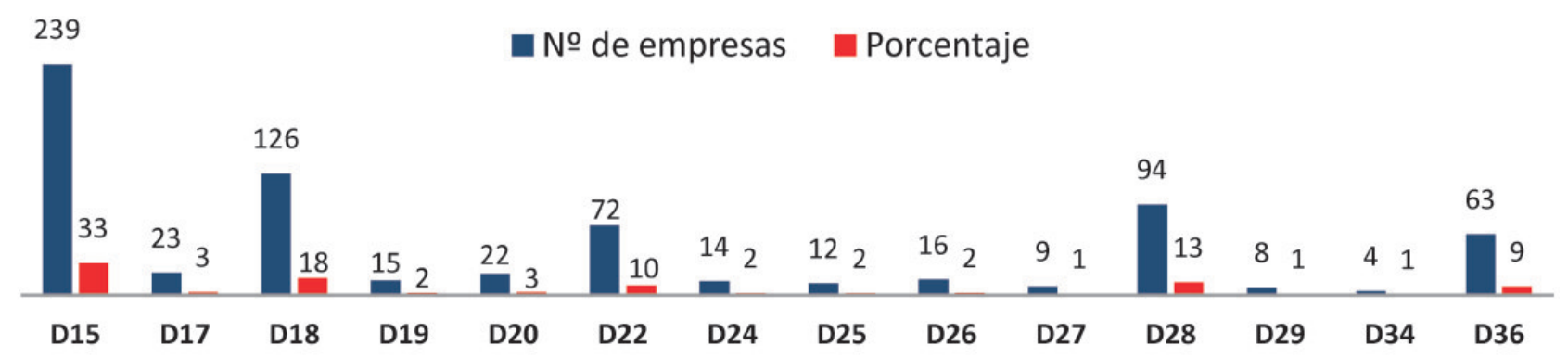

Figura 4. Número de empresas en cada subsector del sector $D$ Industrias manufactureras (CIIU dos dígitos) 
Tabla 1. Empresas en cada subsector del Sector $D$ Industrias manufactureras (CIIU dos dígitos)

\begin{tabular}{|c|c|c|c|}
\hline $\begin{array}{l}\text { Código } \\
\text { CIIU }\end{array}$ & Descripción & $\begin{array}{l}\text { Número } \\
\text { empresas }\end{array}$ & $\begin{array}{c}\text { Porcentaje } \\
\text { empresas por código }\end{array}$ \\
\hline D15 & Elaboración de productos alimenticios y de bebidas & 239 & 33 \\
\hline D17 & Fabricación de productos textiles & 23 & 3 \\
\hline D18 & Fabricación de prendas de vestir; preparado y teñido de pieles & 126 & 18 \\
\hline D19 & $\begin{array}{l}\text { Curtido y preparado de cueros; fabricación de calzado; fabricación de } \\
\text { artículos de viaje, maletas, bolsos de mano y similares; artículos }\end{array}$ & & \\
\hline \multirow{3}{*}{ D20 } & de talabartería y guarnicionería & 15 & 2 \\
\hline & transformación de la madera y fabricación de productos de madera y de corcho, & & \\
\hline & excepto muebles; fabricación de artículos de cestería y espartera & 22 & 3 \\
\hline D22 & Actividades de edición e impresión y de reproducción de grabaciones & 72 & 10 \\
\hline D24 & Fabricación de sustancias y productos químicos & 14 & 2 \\
\hline D25 & Fabricación de productos de caucho y de plástico & 12 & 2 \\
\hline D26 & Fabricación de otros productos minerales no metálicos & 16 & 2 \\
\hline D27 & Fabricación de productos metalúrgicos básicos & 9 & 1 \\
\hline D28 & Fabricación de productos elaborados de metal, excepto maquinaria y equipo & 94 & 13 \\
\hline D29 & Fabricación de maquinaria y equipo ncp & 8 & 1 \\
\hline D34 & Fabricación de vehículos automotores, remolques y semirremolques & 4 & 1 \\
\hline D36 & Fabricación de muebles; industrias manufactureras ncp & 63 & 9 \\
\hline & TOTAL & 718 & 100 \\
\hline
\end{tabular}

Se procedió a establecer una muestra que permitiera seleccionar al azar un número de empresas que aportaran los datos necesarios para llevar a cabo la investigación confiable. Teniendo en cuenta un nivel de confianza del $95 \%$, un error máximo permitido del $5 \%$ y una población total de 729 de los sectores A,C y D la muestra corresponde a 171 empresas a encuestar (ver tabla 2). La selección del número de encuestas por cada sector se realizo mediante pro rateo, lo que implica que los sectores que aglutinan mayor número de empresas serán aquellos donde se realicen mayor número de encuestas (ver tabla 3).
Tabla 2. Tamaño de la muestra

$\begin{array}{lr}\text { Población } & 718,00 \\ \text { Nivel de confianza (95\%) } & 1,65 \\ \text { Error }(5 \%) & 0,055 \\ \text { p } & 0,5 \\ q & 0,5 \\ \text { Tamaño de la muestra } & 225 \\ \text { Muestra corregida } & 171\end{array}$

Tabla 3. Total empresas encuestadas por sectores

\begin{tabular}{|c|c|c|c|c|c|}
\hline $\begin{array}{l}\text { Código } \\
\text { CIIU }\end{array}$ & Descripción & $\begin{array}{l}\text { Núm. } \\
\text { empresas }\end{array}$ & $\begin{array}{c}\text { Porcentaje } \\
\text { empresas } \\
\text { por códigos }\end{array}$ & $\begin{array}{c}\text { Núm. } \\
\text { de empresas } \\
\text { encuestadas }\end{array}$ & $\begin{array}{l}\text { Porcentaje } \\
\text { de empresas } \\
\text { por sector }\end{array}$ \\
\hline A & Agricultura, ganadería, caza y silvicultura & 12 & 1,63 & 1 & 0,60 \\
\hline C & Explotación de minas y canteras & 9 & 1,22 & 4 & 2,41 \\
\hline D & Industrias manufactureras & 717 & 97,15 & 161 & 96,99 \\
\hline & Total & 738 & 100 & 166 & 100,00 \\
\hline
\end{tabular}


En resumen, se entrevistaron 184 empresas de las cuales 166 se incluyeron en el estudio. Un 0,60\% se realizaron en el sector A, AGRICULTURA, GANADERÍA, CAZA Y SILVICULTURA, el 2,41\% (4 encuestas) al sector B, EXPLOTACIÓN DE MINAS Y CANTERAS y las restantes al sector $\mathrm{C}$, INDUSTRIAS MANUFACTURERAS, (ver tabla 3).

Para la recolección de los datos se diseñó el formato de encuesta para caracterizar los sistemas de planificación y control de la producción de las pymes de la ciudad de Neiva. Este instrumento se dividió en tres partes fundamentales: la primera los datos generales de la empresa, la segunda se preguntaron los aspectos que tienen que ver con la caracterización del sistema productivo de la unidad industrial y por último una tercera parte que busca identificar que estudios tiene la empresa que le permita conocer sus capacidades reales de equipo, maquinaria y mano de obra y cuáles son los recursos informáticos que utiliza para identificar su capacidad de producción, sus inventarios, sus recursos humanos y la planeación de la producción. La encuesta fue validada inicialmente mediante consulta a especialistas en la Ciudad de Medellín y posteriormente fue aplicada a una muestra de la población a encuestar para verificar el cumplimiento de objetivos.

Se realizaron 284 encuestas distribuida así (ver figura 5), 184 exitosas; 29 empresas no colaboraron con el estudio correspondiente al $11,69 \%$ del total. 16 empresas ya habían dejado de operar, correspondiente al $6,45 \%$. Del total escogido 8 empresas tenían mal registrada la dirección en la Cámara de Comercio de Neiva y fue imposible encontrar la dirección correcta.

Un total de 11 de las empresas escogidas estaban mal codificadas, su objetivo es la comercialización, razón por la cual se detectaron y se eliminaron de la lista inicial.
La clasificación en el cogido CIIU en los sectores A, B, C, $D$ corresponde a empresas que exploten, o manufacturen cualquier tipo de artículo. Corresponden al 4,44\% del total de la muestra.

\section{Resultados del estudio}

En las medianas empresas el $67 \%$ cuenta con estudios de tiempos, con indicadores de capacidad y eficiencia del total de la planta de producción y el $83 \%$ conocen su capacidad de producción, sin embargo solamente el $33 \%$ cuenta con indicadores de eficiencia y utilización para cada centro de trabajo. El 100\% de ellas cuenta con información confiable sobre sus ventas en los últimos 5 años y por lo tanto pronostica las mismas con base en esos registros.

El 83.3\% elabora, evalúa planes de producción y establecen la capacidad requerida para los mismos. El total de ellas cuentan con diagramas de ruta, registros de carga, listado de materiales, establece planes para pedido de materiales y mantiene registros confiables de inventarios; lo que les permite tener a la mano la información necesaria para comprometer con un alto índice de confiabilidad su capacidad de producción y satisfacer la demanda requerida por los clientes con un alto índice de probabilidad de cumplir los compromisos pactados.

Como lo podemos ver en la tabla 4 y en la figura 6 , las medianas empresas que corresponden al $3,26 \%$ del total de las organizaciones que existen en la ciudad de Neiva del sector manufacturero tiene 67 empleados promedio y aporta a la región 1,766 empleos. Cuentan con el 100\% del personal calificado para las funciones de Planeación de la Producción y Control de las mismas, tienen en cuenta y conocen los elementos necesarios para lograr una planeación y un control efectivo de su producción que les

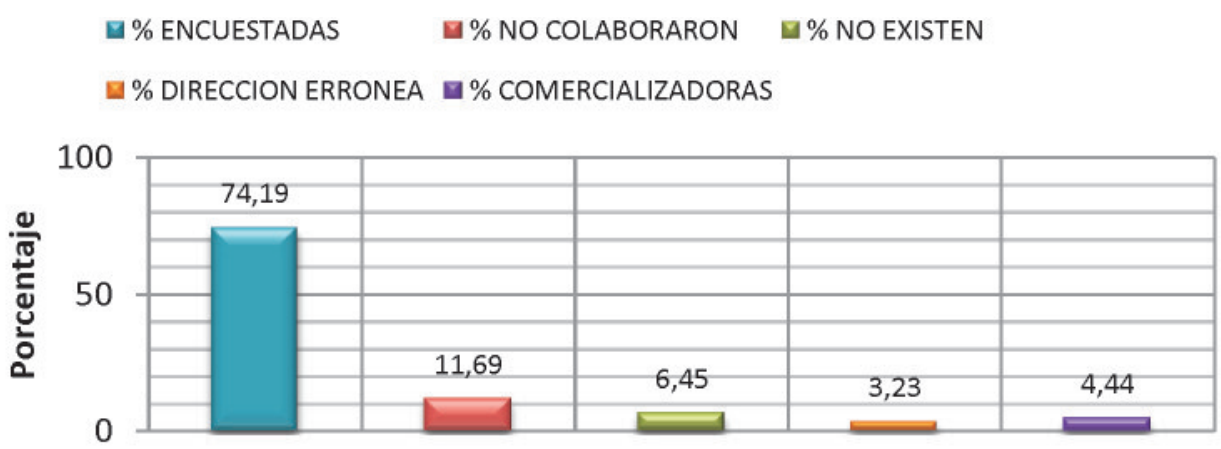

Figura 5. Distribución de las empresas visitadas. 
Tabla 4. Profesión del encargado de planear la producción

\begin{tabular}{|lrrrrrr|}
\hline Descripción & Mediana & \% Mediana & Pequeña & \% Pequeña & Micro & $\%$ Micro \\
\hline Ingeniero Industrial & 3 & 50,00 & 4 & 23,53 & 3 & 1,86 \\
Otras Ingenierías & 2 & 33,33 & 1 & 5,88 & 5 & 3,11 \\
Contador & 0 & 0,00 & 1 & 5,88 & 1 & 0,62 \\
Admón de empresas & 1 & 16,67 & 4 & 23,53 & 8 & 4,97 \\
Técnico Sena & 0 & 0,00 & 0 & 0,00 & 10 & 6,21 \\
Tecnólogo & 0 & 0,00 & 1 & 5,88 & 8 & 4,97 \\
Diseñador & 0 & 0,00 & 0 & 0,00 & 5 & 3,11 \\
Empírico & 0 & 0,00 & 6 & 35,29 & 76 & 47,20 \\
Otros & 0 & 0,00 & 0 & 0,00 & 45 & 27,95 \\
\hline Total & 6 & 100,00 & 17 & 100,00 & 161 & 100,00 \\
\hline
\end{tabular}

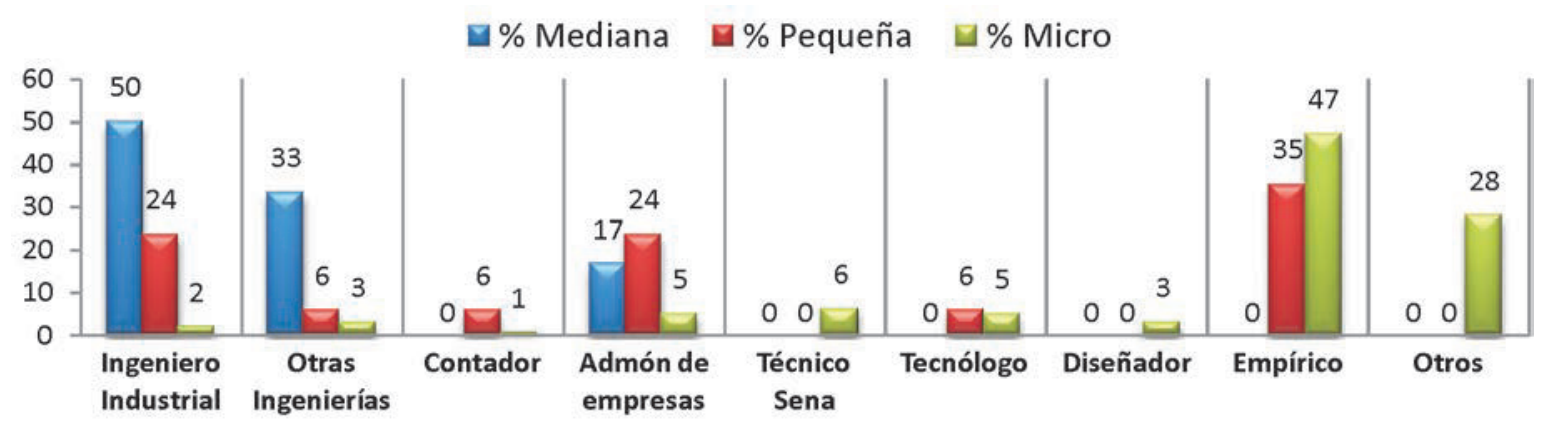

Figura 6. Profesión del encargado de planear la producción.

permite responder ante las fluctuaciones de la demanda y ante las necesidades del cliente, a pesar de que no cuentan con la tecnología adecuada para una planeación más eficiente.

Solamente el $66,67 \%$ de las mediana cuentan con software como recurso tecnológico para el desarrollo de las funciones de planeación (ver tabla 5). La industria manufacturera en general no cuenta con la tecnología de manufactura que les permita mayores volúmenes de producción, no han entrado en la era de la informática aplicada a la manufactura. Tecnologías de manufactura como CAM, CIM no han sido implementadas en las empresas de la región. «La industria manufacturera por estar estrechamente ligada al desarrollo de la ciencia, la tecnología y la ingeniería es un sector económico propenso a crear, experimentar y fomentar dinámicas de aprendizaje y difusión del conocimiento» ${ }^{4}$.
En relación con la pequeña empresa, solamente un $33 \%$ de ellas cuentan con estudios de tiempos y conocen los indicadores de capacidad total. El $23 \%$ conoce los indicadores de capacidad total y de cada uno de los centros de producción. Con respecto a indicadores de utilización solamente el 17\% conoce la rata de utilización de los centros, pero un $29 \%$ conoce la tasa de utilización total de la misma.

La pequeña empresa de la ciudad, aporta en promedio 15 empleos, lo que indica que en total esta contribuye con aproximadamente 1110 empleos (ver tablas 6 y 7 ), contando con el $64,7 \%$ de personal calificado para las labores de planificación y control de la producción como lo muestra la tabla 4.

Con relación a los registros sobre ventas tan solo el $79 \%$ cuenta con estos para un periodo de 5 años y el 52,94

4. Durán Peralta Julián. APRENDIZAJE PRODUCTIVO EN LA INDUSTRIA MANUFACTURERA DE COLOMBIA. UN ESTUDIO A NIVEL DE SECTORES. Publicado en: www. Cuadernos de economía. 29(52):2010. 
Tabla 5. Recursos tecnológicos para planeación

\begin{tabular}{lccccccccccccc|}
\hline Recursos informáticos & \multicolumn{4}{c}{ Mediana empresa } & \multicolumn{4}{c|}{ Pequeña empresa } & \multicolumn{4}{c|}{ Micro empresa } \\
& Si & $\%$ & No & $\%$ & Si & $\%$ & No & $\%$ & Si & $\%$ & No & $\%$ \\
\hline $\begin{array}{l}\text { Software de inventarios } \\
\text { Software para planear }\end{array}$ & 4 & 67 & 2 & 33 & 4 & 24 & 13 & 76 & 9 & 6 & 152 & 94 \\
la producción & 2 & 33 & 4 & 67 & 1 & 6 & 16 & 94 & 5 & 3 & 156 & 97 \\
Software para pronósticos & 2 & 33 & 4 & 67 & 3 & 18 & 14 & 82 & 5 & 3 & 156 & 97 \\
\hline
\end{tabular}

Tabla 6. Clasificación de las pymes y distribución de los empleos

\begin{tabular}{lcccccc} 
PYMES & $\begin{array}{c}\text { Núm. de } \\
\text { empresas }\end{array}$ & $\begin{array}{c}\text { Porcentaje } \\
\text { Núm. empresas empleados }\end{array}$ & $\begin{array}{c}\text { Total } \\
\text { Total empleos }\end{array}$ empleados \\
\hline Mediana $(51-200)$ & 6 & 3,26 & 400 & 33,17 & 67 \\
Pequeña $(10-50)$ & 17 & 9,24 & 262 & 21,72 & 15 & 3 \\
Micro(1 - 10) & 161 & 87,50 & 544 & 45,11 & 85 \\
\hline Total & 184 & 100,00 & 1206 & 100,00 & 85 \\
\hline
\end{tabular}

Tabla 7. Proyección y distribución de los empleos de las Mipymes

\begin{tabular}{lcc} 
MIPYMES & $\begin{array}{c}\text { Núm. de } \\
\text { empresas }\end{array}$ & $\begin{array}{c}\text { Total } \\
\text { empleados }\end{array}$ \\
\hline Mediana $(51-200)$ & 26 & 1733 \\
Pequeña $(10-50)$ & 72 & 1110 \\
Micro $(1-10)$ & 683 & 2308 \\
\hline Total & 781 & 5151 \\
\hline
\end{tabular}

los utiliza para pronosticar sus ventas. La pequeña empresa no elabora planes maestros y por lo tanto no los puede evaluar. En otros indicadores estudiados como la identificación de la capacidad necesaria para cada lote, solamente el 17,24\% la determinan, el 29,41 de las empresas cuentan con los diagramas de ruta de sus productos y el $35,29 \%$ cuenta con registros de carga para cada operación. Podemos notar entonces que hay un significativo deterioro de los mismos con respecto a la mediana empresa. Sin embargo los demás indicadores como el listado de materiales por producto $(52,94 \%)$, el registro para identificar cuanto pedir $(41,18 \%)$ y el registro de inventarios $(64 \%)$ cuentan con registros confiables.
Frente a los recursos tecnológicos se evidencia también la falta de utilización del software para la elaboración de planes agregados, maestros y requerimiento de materiales, lo mismo que de sistemas para pronosticar las ventas (ver figura 7).

Como podemos observar en la figura 8 las nuevas tecnologías de la producción apoyadas en los sistemas informáticos están más desarrolladas en la pequeña empresa que en la mediana. La razón básica es que la tecnología CAD implementada en la industria gráfica por razones de costo ha permitido que esta vaya adquiriendo importancia. Las tecnologías de la manufactura están siendo mejor adaptadas en la pequeñas empresas.

Los datos arrojados por el estudio con referencia a la micro empresa son por demás desalentadores: el 9,5\% ha realizado o tiene un estudio de tiempos, el 10,6\% conoce su capacidad de producción, solamente el 4,35\% tiene indicadores de eficiencia de sus centros y de la planta en general. Lo mismo ocurre con el indicador de utilización, un $3,73 \%$ conoce el indicador de cada centro y el $4,35 \%$ el de la utilización de la planta en general. El $44,1 \%$ tiene registros de ventas y solamente el $18 \%$ se apoya en ellos para pronosticar sus ventas. 


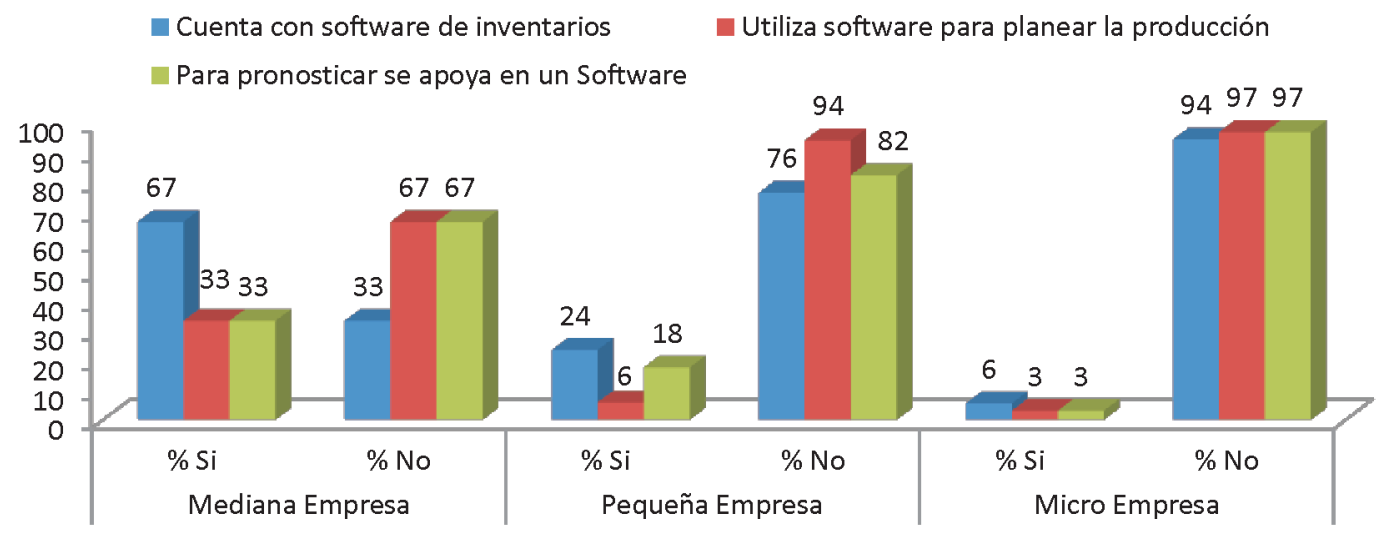

Figura 7. Recursos tecnológicos para planeación.

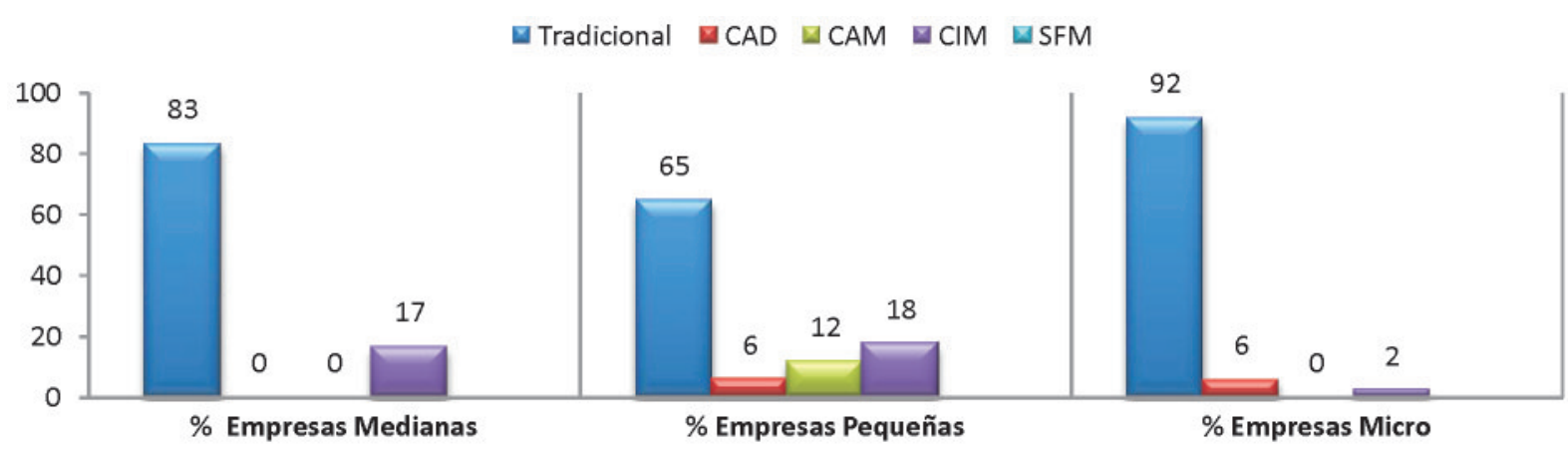

Figura 8. Clasificación de los sistemas productivos según la tecnología de la producción.

El $3,11 \%$ de las microempresas de la ciudad elaboran plan maestro para su producción, el 0,62\% lo evalúa, el $2,48 \%$ identifica la capacidad necesaria de los recursos para cada lote, el $8 \%$ cuenta con los diagramas de rutas de sus procesos y el $4,98 \%$ lleva registros de car- gas de cada centro. El 23,6\% cuenta con el listado de materiales de sus productos, el $16,77 \%$ con registros para establecer cuanto pedir y solamente el $27,95 \%$ mantiene registro de sus inventarios (ver tablas 8 y 9, y figuras 9 y 10).

Tabla 8. Elementos de planeación a mediano plazo

\begin{tabular}{|c|c|c|c|c|c|c|c|c|c|c|c|c|}
\hline \multirow[t]{2}{*}{ Elementos } & \multicolumn{4}{|c|}{ Mediana empresa } & \multicolumn{4}{|c|}{ Pequeña empresa } & \multicolumn{4}{|c|}{ Micro empresa } \\
\hline & $\mathrm{Si}$ & $\% \mathrm{Si}$ & No & $\%$ No & Si & $\% \mathrm{Si}$ & No & $\%$ No & Si & $\% \mathrm{Si}$ & No & $\%$ No \\
\hline Estudios de tiempos & 4 & 66,67 & 2 & 33,33 & 6 & 35,29 & 11 & 64,71 & 15 & 9,32 & 146 & 90,68 \\
\hline $\begin{array}{l}\text { Capacidad de producción } \\
\text { Eficiencia de cada centro }\end{array}$ & 5 & 83,33 & 1 & 16,67 & 6 & 35,29 & 11 & 64,71 & 17 & 10,56 & 144 & 89,44 \\
\hline de producción & 3 & 50,00 & 3 & 50,00 & 4 & 23,53 & 13 & 76,47 & 7 & 4,35 & 154 & 95,65 \\
\hline Eficiencia del total de la planta & 4 & 66,67 & 2 & 33,33 & 4 & 23,53 & 13 & 76,47 & 7 & 4,35 & 154 & 95,65 \\
\hline Utilización de cada centro & 2 & 33,33 & 4 & 66,67 & 3 & 17,65 & 14 & 82,35 & 6 & 3,73 & 155 & 96,27 \\
\hline Utilización total de la planta & 4 & 66,67 & 2 & 33,33 & 5 & 29,41 & 12 & 70,59 & 7 & 4,35 & 154 & 95,65 \\
\hline Tiene registros de ventas & & & & & & & & & & & & \\
\hline $\begin{array}{l}\text { de los últimos } 5 \text { años } \\
\text { Pronostica el tamaño }\end{array}$ & 6 & 100,00 & 0 & 0,00 & 12 & 70,59 & 5 & 29,41 & 71 & 44,10 & 90 & 55,90 \\
\hline de las ventas & 6 & 100,00 & 0 & 0,00 & 9 & 52,94 & 8 & 47,06 & 29 & 18,01 & 132 & 81,99 \\
\hline
\end{tabular}


A pesar de la necesidad de ponernos a tono con el mercado internacional, con la herramienta utilizada por todos los paises del mundo las ISO, en la región solamente el $33 \%$ de las medianas empresas, el $17,65 \%$ de las pequeñas y el 1,24 de las micro se han acogido a este mecanismo. El $100 \%$ de las empresas, medianas, pequeñas y micros no han tenido conciencia ambiental o por lo menos no lo han demostrado asumiendo los estándares de gestión ambiental resumidos en las normas Iso 14.000 y las normas Oshas 18.000 de la Salud ocupacional (ver tabla $10 \mathrm{y}$ figuras 11).
Las Certificadoras han hecho esfuerzos para que las mipymes se acojan a los planes de gestión de calidad, ambiental y de salud ocupacional que les permita insertarse en los mercados nacionales e internacionales y han rebajado las condiciones mínimas necesarias en especial las que tienen que ver con la administración de las mismas. Sin embargo la implementación y el sostenimiento de las mismas son onerosas para la mayoría de las empresas de la región, en razón a su tamaño.
Estudios de tiempos

n Eficiencia del total de la planta

플 Tiene registros de ventas de los ultimos 5 años

\section{- Capacidad de producción}

- Utilización de cada centro

Eronostica el tamaño de las ventas
Eficiencia de cada centro de producción

- Utilización total de la planta

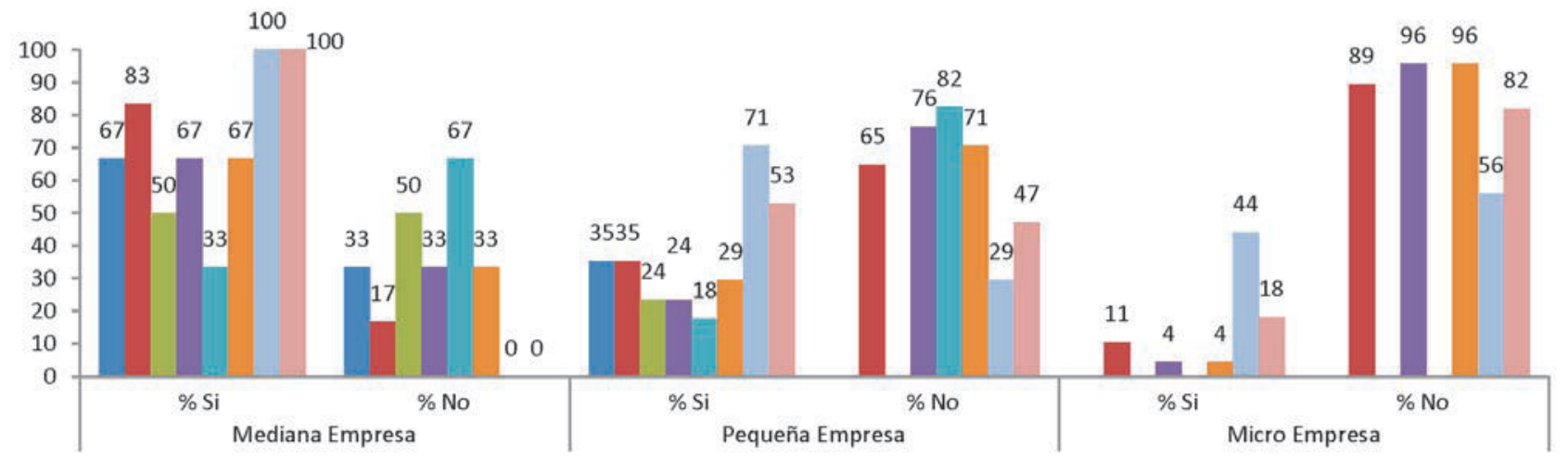

Figura 9. Elementos de planeación a mediano plazo.

Establece Plan Maestro

- Establece la capacidad necesaria de cada lote

- Cuenta con registros de carga de cada operación

n Cuenta con registro para identificar cuando pedir
- Evalua los diferentes planes

- Cuenta con diagramas de ruta para sus productos - Cuenta con lista de materiales para cada producto Cuenta con registro de inventarios

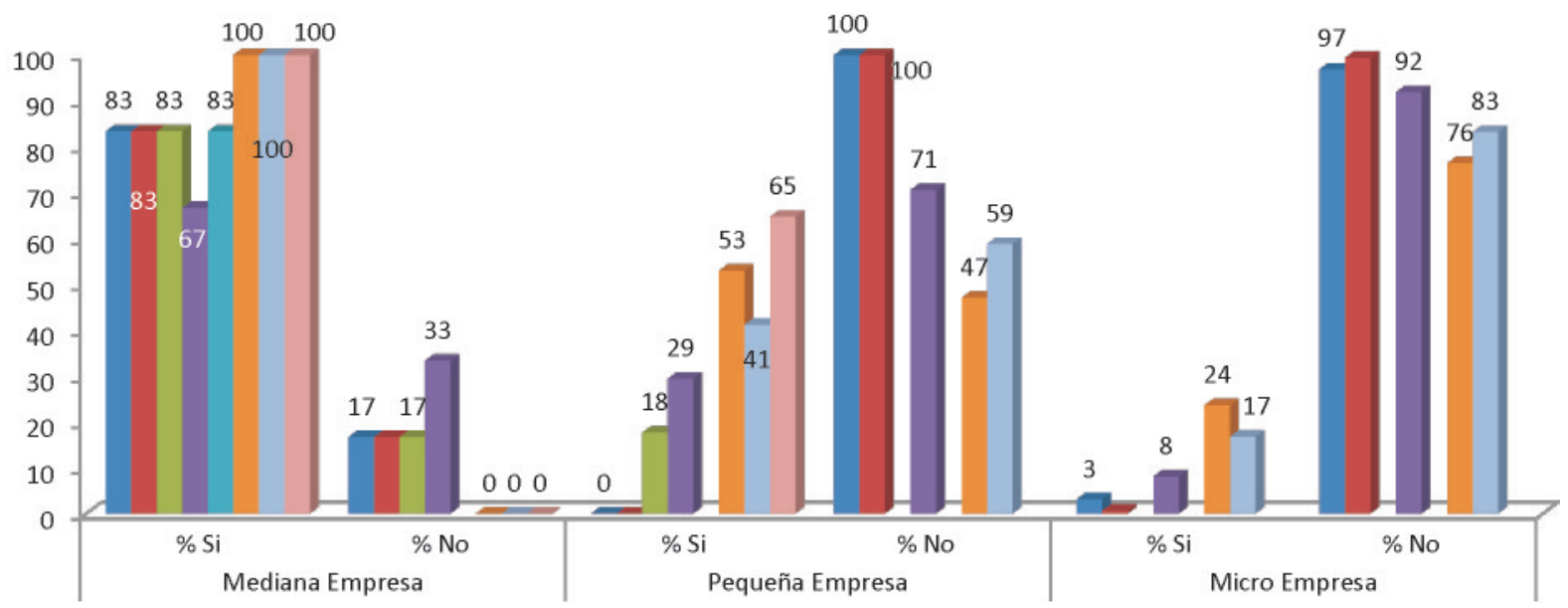

Figura 10. Elementos de planeación a corto plazo. 
Tabla 9. Elementos de planeación a corto plazo.

\begin{tabular}{|c|c|c|c|c|c|c|c|c|c|c|c|c|}
\hline \multirow[t]{2}{*}{ Elemento } & \multicolumn{4}{|c|}{ Mediana empresa } & \multicolumn{4}{|c|}{ Pequeña empresa } & \multicolumn{4}{|c|}{ Micro empresa } \\
\hline & Si & $\% \mathrm{Si}$ & No & $\%$ No & Si & $\% \mathrm{Si}$ & No & $\%$ No & Si & $\% \mathrm{Si}$ & No & $\%$ No \\
\hline Establece plan maestro & 5 & 83,33 & 1 & 16,67 & 0 & 0 & 17 & 100 & 5 & 3,11 & 156 & 96,89 \\
\hline $\begin{array}{l}\text { Evalúa los diferentes planes } \\
\text { Establece la capacidad }\end{array}$ & 5 & 83,33 & 1 & 16,67 & 0 & 0 & 17 & 100 & 1 & 0,62 & 160 & 99,38 \\
\hline $\begin{array}{l}\text { necesaria de cada lote } \\
\text { Cuenta con diagramas }\end{array}$ & 5 & 83,33 & 1 & 16,67 & 3 & 17,65 & 14 & 82,35 & 4 & 2,48 & 157 & 97,52 \\
\hline $\begin{array}{l}\text { de ruta para sus productos } \\
\text { Cuenta con registros }\end{array}$ & 4 & 66,67 & 2 & 33,33 & 5 & 29,41 & 12 & 70,59 & 13 & 8,07 & 148 & 91,93 \\
\hline $\begin{array}{l}\text { de carga de cada operación } \\
\text { Cuenta con lista de materiales }\end{array}$ & 5 & 83,33 & 1 & 16,67 & 6 & 35,29 & 11 & 64,71 & 8 & 4,97 & 153 & 95,03 \\
\hline $\begin{array}{l}\text { para cada producto } \\
\text { Cuenta con registro para }\end{array}$ & 6 & 100,00 & 0 & 0,00 & 9 & 52,94 & 8 & 47,06 & 38 & 23,60 & 123 & 76,40 \\
\hline $\begin{array}{l}\text { identificar cuando pedir } \\
\text { Cuenta con registro }\end{array}$ & 6 & 100,00 & 0 & 0,00 & 7 & 41,18 & 10 & 58,82 & 27 & 16,77 & 134 & 83,23 \\
\hline de inventarios & 6 & 100,00 & 0 & 0,00 & 11 & 64,71 & 6 & 35,29 & 45 & 27,95 & 116 & 72,05 \\
\hline
\end{tabular}

Tabla 10. Sistemas de gestión integral.

\begin{tabular}{|c|c|c|c|c|c|c|c|c|c|c|c|c|}
\hline \multirow{2}{*}{$\begin{array}{l}\text { MIPYME } \\
\text { Descripción }\end{array}$} & \multicolumn{4}{|c|}{$\begin{array}{c}\text { Sistema de gestión } \\
\text { de calidad }\end{array}$} & \multicolumn{4}{|c|}{$\begin{array}{c}\text { Sistema de gestión } \\
\text { ambiental }\end{array}$} & \multicolumn{4}{|c|}{$\begin{array}{c}\text { Sistema de gestión } \\
\text { so }\end{array}$} \\
\hline & SI & $\% \mathrm{SI}$ & NO & $\%$ NO & SI & $\% \mathrm{SI}$ & NO & $\%$ NO & SI & $\% \mathrm{SI}$ & NO & $\%$ NO \\
\hline Mediana & 2 & 33,33 & 4 & 66,67 & 0 & 0,00 & 6 & 100,00 & 1 & 16,67 & 5 & 83,33 \\
\hline Pequeña & 3 & 17,65 & 14 & 82,35 & 1 & 5,88 & 16 & 94,12 & 2 & 11,76 & 15 & 88,24 \\
\hline Micro & 2 & 1,24 & 159 & 98,76 & 0 & 0,00 & 161 & 100,00 & 0 & 0,00 & 161 & 100,00 \\
\hline Total & 7 & 3,80 & 177 & 96,20 & 1 & 0,54 & 183 & 99,46 & 3 & 1,63 & 181 & 98,37 \\
\hline
\end{tabular}

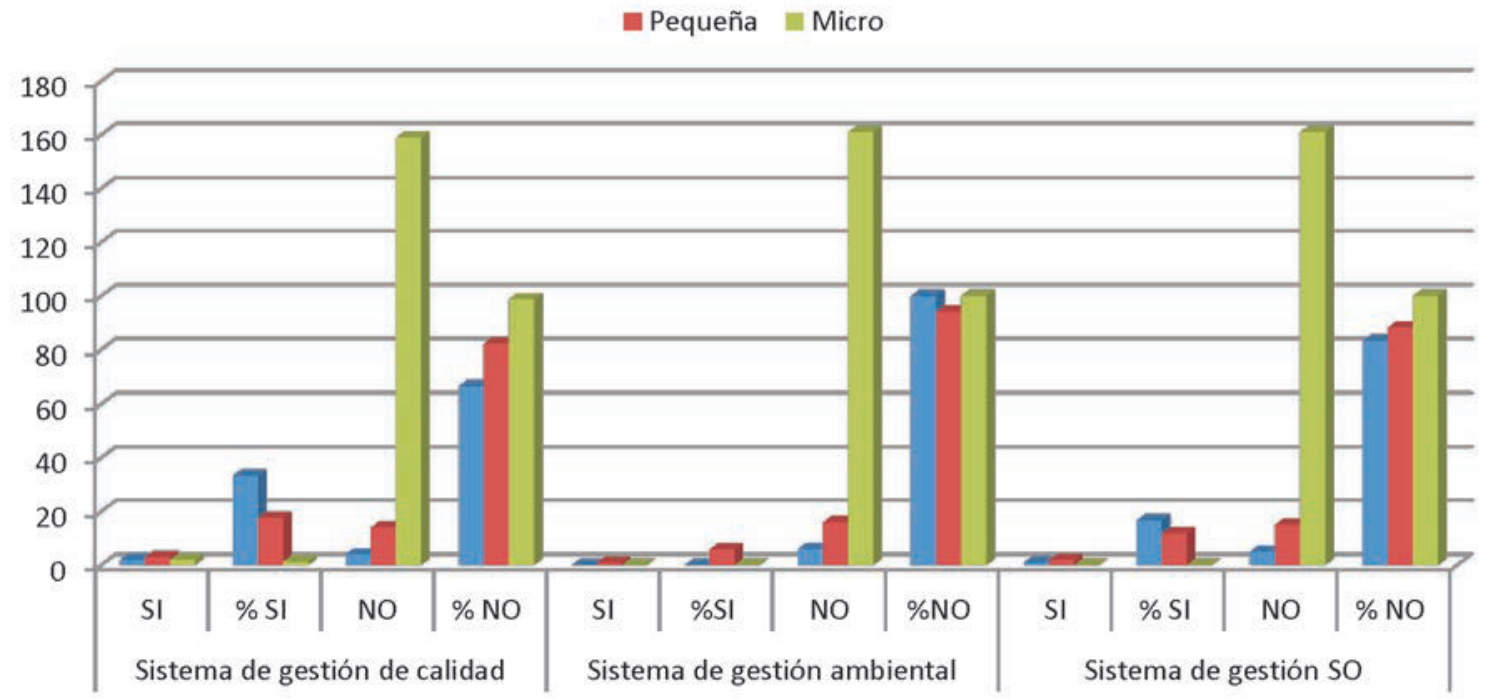

Figura 10. Sistemas de gestión integral. 


\section{Conclusiones}

Las empresas no tienen la cultura de la necesidad de conocerse; conocer su proceso productivo, ni los tiempos de fabricación, ni los procedimientos más adecuados para la fabricación de sus productos, por lo que no le permite tener registros históricos del comportamiento de sus ventas, eficiencias, inventarios, costos, que le proporcionarían los elementos necesarios para elaborar sus planes de producción y ventas adecuadamente, permitiéndole mejor utilización de su capacidad productiva y por ende poder comprometerse con mayores volúmenes de ventas, que le traerían como consecuencia los recursos necesarios para el cambio de tecnologías de manufactura que le permitirá atender nuevos mercados, incrementar su plantilla de mano de obra, mejorar calidad de vida de los ciudadanos y un desarrollo industrial de la ciudad.

El estudio arroja como resultado que las pequeñas empresas y las micro empresas, carecen de sistemas de planeación y control y que los registros históricos necesarios para adelantar dichas labores son por demás incipientes. Es factible que los propietarios sepan o conozcan los datos pero no hay registros ciertos que puedan ser utilizados para desarrollar sistemas de planeación confiables en las organizaciones.

La utilización de la tecnología informática y la tecnología de fabricación podemos clasificarla como nula. Los sistemas CAD son los únicos utilizados por algunas empresas, en especial la industria gráfica, para la elaboración de los diseños de los impresos que elaboran para las empresas de la región.

La redefinición de una política industrial para la ciudad que cuenta con una economía en transformación y con la permanencia de grandes problemas en el sector de la industria manufacturera, como lo ha demostrado este estudio, con Cluster no muy robustos, donde hemos visto que hay bajos niveles de productividad, volúmenes de exportación insignificantes comparados con los de otras ciudades, es de vital importancia.

La región no cuenta, como se percibió a lo largo del estudio, con mano de obra que tenga cultura empresarial, priman en el trabajador los modelos tradicionales de no trabajo en las horas pico, la siesta sigue siendo un factor determinante en el rendimiento del empleado, el no trabajo por turnos en las labores industriales, la falta de compromiso del trabajador para aportar su trabajo en momentos que lo requiera la empresa, son la queja permanente de los empresarios, en especial los de otras zonas del país.

La falta de mano de obra calificada para trabajos de manufactura, a pesar de los esfuerzos hechos por el Sena; la falta de técnicos y tecnólogos en las diferentes disciplinas u oficios manufactureras unido a la cultura del querer ser trabajador petrolero, han hecho que la industria manufacturera no tenga el auge que requiere un departamento que dice tener una vocación agroindustrial.

Los sectores más desarrollados a nivel de la manufactura son: la fabricación de productos elaborados de metal, excepto maquinaria y equipo con un $13 \%$ de las industrias del sector; la fabricación de muebles; industrias manufactureras con un 9\%; las actividades de edición e impresión y de reproducción de grabaciones con un $10 \%$ donde está más desarrollada la tecnología manufacturera del CAD; la fabricación de prendas de vestir; preparado y teñido de pieles con un 18\%; elaboración de productos alimenticios y de bebidas con un $33 \%$. Ninguna de las empresas tiene presencia a nivel nacional.

\section{Recomendaciones}

Ante las conclusiones arrojadas por el estudio podemos hacer las siguientes recomendaciones:

Es necesario acercar, como lo propone el Informe de la Comisión de sabios de la Misión de Ciencia Educación y Desarrollo $0^{5}$, a la universidad y a la empresa; a la empresa como motor de desarrollo y a la universidad como proveedora de ciencia y tecnología.

Capacitar al recurso humano del sector manufacturero de la ciudad para cambiar las estructuras culturales de la región con el fin de generar mayor simbiosis entre las acciones para la competitividad y el recurso humano necesario para el mejoramiento.

La Universidad debe lograr un mayor acercamiento entre la empresa y el Estado para permitir que los recursos del estado destinados para el mejoramiento de los sistemas productivos de las organizaciones empresariales de la ciudad, se vean representados en una mayor cobertura de la demanda y una mayor proyección de la industria de la ciudad en los mercados nacionales e internacionales. 
Es necesario además, buscar caminos que nos permitan lograr mayores niveles de asociatividad entre los empresarios de la región para la obtención de mejores precios de compra de las materias primas, mejor utilización de los canales de comercialización, disminución de los costos de fabricación en razón a una mayor y mejor utilización de los recursos de las empresas.

Diseñar campañas que no tengan como objetivo mover el corazón de los compradores con base en falsos nacionalismos, si no pensar que el comprador no tiene corazón, compra con la cabeza, busca lo más barato, de la mejor calidad sin importar quien se lo proporcione.

\section{Referencias bibliográficas}

Alfalla Luque Rafaela, García Sánchez Mㅡㅁosa, y otros. Introducción a la dirección de operaciones táctico-operativa: un enfoque práctico. España: Editorial DELTA, 2008.

Bello Pérez Carlos José. Manual de producción aplicado a las pyme. Ecoe ediciones, 2006.

Cuatrecasas Luis. Diseño avanzado de procesos y plantas de producción flexible. España: Editorial Profit, 2009.

Chapman Stephen N. Planificación y control de la producción. Pearson, Prentice Hall, 2006.

Dickeman Allen. Control adecuado de la producción disponible en: http://www.control adecuado de la producción Fecha de consulta junio 2010.

Domínguez Machuca, González Santiago y otros. Dirección de la producción y de operaciones, decisiones tácticas. Prentice Hall, 2008.

Domínguez Machuca, González Santiago y otros. Dirección de operaciones, aspectos estratégicos en la producción y servicios. México: McGraw Hill, 1995.

Gaither Norman, Frazier Greg. Administración de producción y operaciones. International Thomson, 2000.

Jay Heizer, Barry Render. Principios de administración de operaciones. Prentice Hall, 2009.
Krajewski Lee J, Larry P. Ritzman. Administración de operaciones: estrategia y análisis. México: Editorial Pearson Educación, 2000.

Mikell P. Groover. Fundamentos de manufactura moderna: materiales, procesos y sistemas. México: Editorial Pearson, 1997.

Sábato Jorge y Botana Natalio. La ciencia y la tecnología en el desarrollo futuro de América Latina. Buenos Aires, 1969, pp. 3 y 4.

Sipper Daniel, Robert Bulfin Jr. Planeación y control de la producción, 1998.

Velázquez Mastretta. Administración de los sistemas de producción. México: Editorial Limusa, 2006.

Durán Peralta Julián. Aprendizaje productivo en la industria manufacturera de Colombia; estudio a nivel de sectores. Publicado en www. Cuadernos de economía. 29(52)(2010):41.

Informe de la misión de sabios Colombia al filo de la oportunidad. Consejería presidencial para el desarrollo de la ciencia y Colciencias. Tercer Mundo Editores, 1996.

Negociación de salario mínimo; ACOPI Regional Bogotá. Fecha de consulta: abril 2007. Disponible en: http:// www. acopibogota.org.co

Plan regional de competitividad del departamento del Huila, comisión regional de competitividad y productividad del Huila 2010. Oficina de Competitividad y productividad. Disponible en www.Gobernaciondelhuila,gov.co

Súper sociedades Mapa de Oportunidades del Huila 2011. Disponible en www.supersociedades.gov.co

PYME: Balance 2005 - Perspectivas 2006 - TLC - Negociación de salario mínimo. Acopi Regional Bogotá. Fecha de consulta: abril 2007. Disponible en: http://www. acopibogota.org.co

Informe MOVISOC 2010. Cámara de Comercio de Neiva. Disponible en www.ccneina.org.

5. Informe de la Misión de sabios Colombia al filo de la oportunidad. Consejería presidencial para el desarrollo de la ciencia y Colciencias. Tercer Mundo Editores, 1996. 